\title{
Using NeuroML and neuroConstruct to build neuronal network models for multiple simulators Padraig Gleeson*1, Sharon Crook ${ }^{2}$, Volker Steuber ${ }^{1,3}$ and R Angus Silver ${ }^{1}$
}

Address: ${ }^{1}$ Department of Physiology, University College London, London, UK, ${ }^{2}$ Department of Mathematics and Statistics and School of Life Sciences, Arizona State University, Tempe, Arizona, USA and ${ }^{3}$ School of Computer Science, University of Hertfordshire, Hatfield, Herts, UK

Email: Padraig Gleeson* - p.gleeson@ucl.ac.uk

* Corresponding author

from Sixteenth Annual Computational Neuroscience Meeting: CNS*2007

Toronto, Canada. 7-12 July 2007

Published: 6 July 2007

BMC Neuroscience 2007, 8(Suppl 2):PI doi:I0.I|86//47|-2202-8-S2-PI

(C) 2007 Gleeson et al; licensee BioMed Central Ltd.

Increased use of biologically detailed cellular and network models by the wider neuroscience community is hampered by the variety of simulation platforms and programming languages used to create these models. While experimentalists and theoreticians share common concepts for describing these physiological phenomena a framework for specifying models is not in common use. The Neural Open Markup Language project, NeuroML [1,2]http://www.neuroml.org, is an international, collaborative initiative to develop standards to facilitate exchange and encourage greater accessibility of models of neuronal systems.

The standards, which are specified in XML (eXtensible Markup Language), are arranged in Levels, with each subsequent Level increasing the scope of the standards. Level 1 concentrates on neuroanatomical information (MorphML $[2,3]$ ) and metadata. Level 2 allows for the specification of detailed conductance based cell models with realistic channel and synaptic mechanisms specified in ChannelML. Level 3 (NetworkML) describes networks of these cells arranged and connected in three dimensions.

One application which uses these standards is neuroConstruct, which has a graphical interface for building and visualizing detailed 3D network models. neuroConstruct allows the automatic generation of script files for the GENESIS and NEURON simulators, and can be used for replaying and analyzing simulated cell and network behavior. Examples of cell and network models from mul- tiple brain areas will be demonstrated on these two simulators, as will a preliminary implementation of automatic generation of scripts for execution in parallel computing environments.

The combination of these technologies allows the development of more detailed large scale neuronal network models while managing the huge complexity associated with these systems. The latest version of the NeuroML specifications is available at http://www.mor phml.org:8080/NeuroMLValidator and neuroConstruct is freely available by contacting: p.gleeson@ucl.ac.uk.

\section{Acknowledgements}

This work has been funded by the MRC and the Wellcome Trust.

\section{References}

I. Goddard NH, et al.: Towards NeuroML: model description methods for collaborative modelling in neuroscience. Philos Trans R Soc Lond B Biol Sci 200I, 356( I 4 I 2): I209- I228.

2. Crook S, Gleeson P, Howell F, Svitak J, Silver RA: MorphML: Level I of the NeuroML standards for neuronal morphology data and model specification. Neuroinf 2007 in press.

3. Qi W, Crook S: Tools for neuroinformatic data exchange: An XML application for neuronal morphology data. Neurocomp 2004, 58-60:1091-1095. 